\title{
Multiplex proteomics for prediction of major cardiovascular events in type 2 diabetes
}

\author{
Christoph Nowak $^{1}$ • Axel C. Carlsson ${ }^{1,2} \cdot$ Carl Johan Östgren $^{3} \cdot$ Fredrik H. Nyström $^{3} \cdot$ Moudud Alam $^{4}$. \\ Tobias Feldreich $^{5}$ • Johan Sundström ${ }^{2}$. Juan-Jesus Carrero ${ }^{6}$ - Jerzy Leppert ${ }^{7}$. Pär Hedberg ${ }^{7}$. Egil Henriksen ${ }^{7}$. \\ Antonio C. Cordeiro ${ }^{8}$ - Vilmantas Giedraitis ${ }^{9} \cdot$ Lars Lind $^{2} \cdot$ Erik Ingelsson $^{10} \cdot$ Tove Fall $^{2} \cdot$ Johan Ärnlöv $^{1,5}$
}

Received: 19 February 2018 / Accepted: 18 April 2018 / Published online: 24 May 2018

(C) The Author(s) 2018

\begin{abstract}
Aims/hypothesis Multiplex proteomics could improve understanding and risk prediction of major adverse cardiovascular events (MACE) in type 2 diabetes. This study assessed 80 cardiovascular and inflammatory proteins for biomarker discovery and prediction of MACE in type 2 diabetes.

Methods We combined data from six prospective epidemiological studies of 30-77-year-old individuals with type 2 diabetes in whom 80 circulating proteins were measured by proximity extension assay. Multivariable-adjusted Cox regression was used in a discovery/replication design to identify biomarkers for incident MACE. We used gradient-boosted machine learning and lasso regularised Cox regression in a random $75 \%$ training subsample to assess whether adding proteins to risk factors included in the Swedish National Diabetes Register risk model would improve the prediction of MACE in the separate $25 \%$ test subsample. Results Of 1211 adults with type 2 diabetes (32\% women), 211 experienced a MACE over a mean ( \pm SD) of $6.4 \pm 2.3$ years. We replicated associations ( $<5 \%$ false discovery rate) between risk of MACE and eight proteins: matrix metalloproteinase (MMP)12, IL-27 subunit $\alpha$ (IL-27a), kidney injury molecule (KIM)-1, fibroblast growth factor (FGF)-23, protein S100-A12, TNF receptor (TNFR)-1, TNFR-2 and TNF-related apoptosis-inducing ligand receptor (TRAIL-R)2. Addition of the 80-protein assay to established risk factors improved discrimination in the separate test sample from $0.686(95 \%$ CI $0.682,0.689)$ to 0.748 ( $95 \%$ CI $0.746,0.751)$. A sparse model of 20 added proteins achieved a C statistic of 0.747 (95\% CI 0.653, 0.842) in the test sample. Conclusions/interpretation We identified eight protein biomarkers, four of which are novel, for risk of MACE in community residents with type 2 diabetes, and found improved risk prediction by combining multiplex proteomics with an established risk model. Multiprotein arrays could be useful in identifying individuals with type 2 diabetes who are at highest risk of a cardiovascular event.
\end{abstract}

Keywords Biomarkers $\cdot$ Major adverse cardiovascular event $\cdot$ Proteomics $\cdot$ Risk $\cdot$ Type 2 diabetes

Electronic supplementary material The online version of this article (https://doi.org/10.1007/s00125-018-4641-z) contains peer-reviewed but unedited supplementary material, which is available to authorised users.

Johan Ärnlöv

johan.arnlov@ki.se

1 Division of Family Medicine and Primary Care, Department of Neurobiology, Care Sciences and Society (NVS), Karolinska Institutet, Alfred Nobels Allé 23, SE 14183 Huddinge, Sweden

2 Department of Medical Sciences, Uppsala University, Uppsala, Sweden

3 Department of Medical and Health Sciences, Linköping University, Linköping, Sweden

4 School of Technology and Business Studies/Statistics, Dalarna University, Falun, Sweden
5 School of Health and Social Studies, Dalarna University, Falun, Sweden

6 Department of Medical Epidemiology and Biostatistics, Karolinska Institutet, Stockholm, Sweden

7 Centre for Clinical Research, Uppsala University, Västerås, Sweden

8 Department of Hypertension and Nephrology, Dante Pazzanese Institute of Cardiology, São Paulo, Brazil

9 Department of Public Health and Caring Sciences, Geriatrics, Uppsala University, Uppsala, Sweden

10 Department of Medicine, Division of Cardiovascular Medicine, Stanford University School of Medicine, Stanford, CA, USA 


\section{Research in context}

\section{What is already known about this subject?}

- Major adverse cardiovascular events (MACE) are important causes of death and disability in people with type 2 diabetes, but the identification of high-risk individuals is insufficient and effective prevention requires better understanding of the pathophysiology

\section{What is the key question?}

- Can multiplex plasma and serum proteomics discover new biomarkers and improve risk prediction for future MACE in people with type 2 diabetes, in addition to established risk factors?

\section{What are the new findings?}

- We discovered four novel circulating biomarkers for risk of MACE in individuals with type 2 diabetes (protein S100-A12 [also known as EN-RAGE], kidney injury molecule [KIM]-1, TNF-related apoptosis-inducing ligand receptor [TRAIL-R]2 and IL-27a)

- The addition of multiplex proteomics to a guideline-recommended model significantly improved prediction in an independent test sample, with a $\mathrm{C}$ index of 0.748

\section{How might this impact on clinical practice in the foreseeable future?}

- The combined measurement of circulating inflammatory and cardiovascular proteins improves the prediction of myocardial infarction and stroke in adults with type 2 diabetes and suggests clinical utility of proteomic profiling. The identification of individuals with type 2 diabetes who are most likely to benefit from more aggressive cardiovascular prevention may be improved by adding targeted proteomics to established risk factors

\section{Abbreviations}

CARDIPP

Cardiovascular Risk Factors in Patients with Diabetes: a Prospective Study in Primary Care

EN-RAGE Extracellular newly identified RAGE-binding protein

FDR False discovery rate

FGF Fibroblast growth factor

GBM Gradient-boosted machine

KIM Kidney injury molecule

MACE Major adverse cardiovascular event/s

MIVC Malnutrition, Inflammation and Vascular Calcification

MMP Matrix metalloproteinase

NDR National Diabetes Register

PADVa Peripheral Arterial Disease in Västmanland

PIVUS Prospective Investigation of the Vasculature in Uppsala Seniors

RAGE Receptor of advanced glycation end products

SAVa Study of Atherosclerosis in Västmanland

TNFR TNF receptor

TRAIL TNF-related apoptosis-inducing ligand

TRAIL-R TNF-related apoptosis-inducing ligand receptor

ULSAM Uppsala Longitudinal Study of Adult Men

VaMIS Västmanland Myocardial Infarction Study

\section{Introduction}

The prevalence of type 2 diabetes is increasing worldwide, with currently over 400 million individuals diagnosed and over 190 million undiagnosed as having diabetes [1]. Up to $40 \%$ of the US population will develop type 2 diabetes during their lifetime, and type 2 diabetes is an important contributor to major adverse cardiovascular events (MACE) such as myocardial infarction and stroke - the leading causes of morbidity and mortality in Western countries [2]. Diabetes is one of the strongest risk factors for MACE [3], and one major treatment goal in type 2 diabetes is to prevent MACE. However, compared with those without diabetes, most individuals with type 2 diabetes remain at increased risk of MACE despite optimal treatment according to current guidelines [4, 5]. Cardiovascular prevention is further complicated by increased rates of drug side effects in people with diabetes, including potential adverse glycaemic effects of lipid-modifying agents $[6,7]$.

Newer glucose-lowering drugs such as sodium-glucose co-transporter 2 inhibitors, and cholesterol-modifying agents such as proprotein convertase subtilisin/kexin type 9 (PCSK9) inhibitors, in addition to standard treatment reduce cardiovascular risk in high-risk individuals $[8,9]$. The high treatment costs and potential side effects, however, currently prohibit their prescription in the majority of individuals with diabetes 
$[7,10,11]$. Identification of high-risk individuals in whom the benefits of aggressive prevention outweigh the costs and side effects is therefore crucial. Clinical decision-making based on overall cardiovascular risk in addition to individual risk factors can improve outcomes, as demonstrated for antihypertensive treatment [12]. Available risk models for MACE in type 2 diabetes are, however, only moderately accurate $[13,14]$, and there is a need for better prediction tools to guide healthcare.

Measuring circulating proteins with presumed roles in cardiovascular pathology by targeted proteomics is a promising approach for biomarker discovery [15]. The translation of proteomics into the clinic, however, has so far been hampered by the resource-demanding technology. Multiplex protein arrays that rely on common methods such as PCR, require small sample volumes and are available at a fraction of the cost of large-scale platforms may provide a clinically applicable method for individualised treatment based on biomarker profiles. One such technique, the proximity extension assay, has been shown to be useful for biomarker discovery in cardiometabolic disease [16-18]. Multiprotein assays have been used to discover new risk markers for cardiovascular disease in type 2 diabetes [19], but the proximity extension method has not been tested to predict risk of MACE in type 2 diabetes.

Here, we used a proximity extension assay to measure the abundance of 80 cardiovascular and inflammatory proteins in plasma and serum from six prospective community cohorts of middle-aged people (30-77 years of age) with type 2 diabetes. We aimed to identify markers of future risk of MACE and to assess the assay's performance against an established risk model in the Swedish National Diabetes Register for the prediction of MACE.

\section{Methods}

\section{Participating cohorts}

Cardiovascular Risk Factors in Patients with Diabetes: a Prospective Study in Primary Care The study Cardiovascular Risk Factors in Patients with Diabetes: a Prospective Study in Primary Care (CARDIPP; ClinicalTrials.gov NCT01049737) [20] recruited outpatients aged 55-65 years with type 2 diabetes from 25 primary healthcare centres in the counties of Östergötland and Jönköping, Sweden, between November 2005 and December 2008. Counties were selected to represent different demographic, rural and urban, small- and largeintake areas. Specialist diabetes nurses performed annual assessments [20]. Out of 761 consecutively enrolled participants, 708 with available outcome data and plasma samples were included in the present investigation.

Prospective Investigation of the Vasculature in Uppsala Seniors In 2001, a non-selective sample of Uppsala community residents aged 70 years were recruited to participate in the longitudinal Prospective Investigation of the Vasculature in Uppsala Seniors (PIVUS) study [21] to evaluate measures of endothelial function; 1016 (50.2\%) out of 2025 invited individuals enrolled. Follow-up biomedical assessments have been performed at 5 -yearly intervals (for more information, please see www.medsci.uu.se/pivus/). All 98 participants with type 2 diabetes at baseline were included in the present study.

Uppsala Longitudinal Study of Adult Men In 1970, all 2841 male residents of Uppsala county, Sweden, who had been born between 1920 and 1924 were invited to participate in the Uppsala Longitudinal Study of Adult Men (ULSAM) study [22], and 2322 (81.7\%) were enrolled. Health assessments have been performed regularly since then (for details, please see www.pubcare.uu.se/ulsam/), and the current study includes all 86 participants with type 2 diabetes at an assessment age of 77 years.

Study of Atherosclerosis in Västmanland Between November 2005 and May 2011, the Study of Atherosclerosis in Västmanland (SAVa) [23] enrolled a total of 2315 individuals into three cohorts composed of participants with acute myocardial infarction (Västmanland Myocardial Infarction Study [VaMIS]; NCT01452178), participants with peripheral artery disease (Peripheral Arterial Disease in Västmanland [PADVa]; NCT01452165) and matched control individuals (SAVa-control; for more information, please see https://savastudy.se/). The current study uses data and samples from PADVa and SAVacontrol. PADVa recruited consecutive participants referred to the Vascular Ultrasound Laboratory of Västmanland County Hospital, Västerås, Sweden, who fulfilled one of three inclusion criteria: (1) at least mild internal carotid artery stenosis; (2) claudication symptoms with an ankle-brachial pressure index $\leq 0.90$; or (3) claudication symptoms with signs of arterial occlusive disease in the ipsilateral extremity on ultrasound examination. Out of 614 eligible individuals, 452 (73.6\%) enrolled. Control participants $(n=692)$ were recruited from Swedish residents in the Swedish population register who were matched by age, sex and municipality to participants enrolled in VaMIS. The current study includes all 80 individuals in SAVacontrol and 99 in PADVa who were diagnosed with type 2 diabetes at baseline.

Malnutrition, Inflammation and Vascular Calcification cohort The aim of the Malnutrition, Inflammation and Vascular Calcification (MIVC) cohort [24] is to study risk factors in kidney disease. Between March 2010 and March 2013, the study enrolled 300 consecutive outpatients who were not undergoing dialysis with stage 3-5 chronic kidney disease at the Dante Pazzanese Institute of Cardiology, São Paolo, Brazil. The current study includes all 140 participants with type 2 diabetes. 


\section{Ethical permission}

Participants provided written informed consent, and the study was conducted according to the Declaration of Helsinki. Ethical permission was granted by the ethics committees of Linköping University (Dnr. 26-05; CARDIPP), Uppsala University (Dnr. 251/90 and 97/329 for ULSAM; Dnr. 00419 and 2005/M-079 for PIVUS; Dnr. 2005:382 for SAVa/PADVa) and the Dante Pazzanese Institute of Cardiology (São Paolo, Brazil).

\section{Inclusion criteria and outcome definition}

In CARDIPP, MIVC, SAVa-control and PADVa, type 2 diabetes was defined as a physician diagnosis of type 2 diabetes according to national guidelines (at least two separate fasting glucose levels $\geq 7.0 \mathrm{mmol} / \mathrm{l}$, or at least two separate $\mathrm{HbA}_{1 \mathrm{c}}$ concentrations $>48 \mathrm{mmol} / \mathrm{mol}$ [ $>6.5 \%$; in MIVC], or prescription of diabetes medication). In ULSAM, type 2 diabetes was defined as $\mathrm{HbA}_{1 \mathrm{c}}>48 \mathrm{mmol} / \mathrm{mol}(>6.5 \%)$, prescription of diabetic medication or a fasting plasma glucose level $\geq 7.0 \mathrm{mmol} / 1$. In 25 out of 86 participants included in ULSAM, diabetes was diagnosed by elevated fasting glucose alone. In PIVUS, type 2 diabetes was defined as a physician diagnosis, prescription of glucose-lowering medication or a fasting plasma glucose level $\geq 7.0 \mathrm{mmol} / \mathrm{l}$. In the PIVUS group, diabetes was diagnosed by elevated fasting glucose alone in 21 out of the 98 included participants. Individuals without available fasting frozen plasma or serum samples, or with missing outcome data, were excluded. MACE was defined as a new episode of fatal or non-fatal myocardial infarction (I21 in ICD-10; www.who.int/classifications/icd/en/) or fatal/non-fatal stroke (I60-I63), whichever occurred first, and was from obtained from hospital and death register linkage.

\section{Covariate definitions}

To adjust for established risk factors, we selected all variables included in the Swedish National Diabetes Register (NDR) calculator for 5 year risk of MACE in individuals with type 2 diabetes [13]: sex, systolic blood pressure ( $\mathrm{mmHg}$ ), BMI $\left(\mathrm{kg} / \mathrm{m}^{2}\right)$, current smoking, diagnosis of atrial fibrillation, history of myocardial infarction or stroke, $\mathrm{HbA}_{1 \mathrm{c}}(\mathrm{mmol} / \mathrm{mol}$, $\%)$, HDL-cholesterol and total cholesterol ( $\mathrm{mmol} / \mathrm{l})$, duration of type 2 diabetes (days), microalbuminuria (3-30 mg/mmol urinary creatinine) and macroalbuminuria ( $>30 \mathrm{mg} / \mathrm{mmol}$ urinary creatinine). Additional covariates included current antihypertensive, statin or diabetes medication, LDL-cholesterol $(\mathrm{mmol} / \mathrm{l})$ and eGFR $\left(\mathrm{ml} \mathrm{min}^{-1}[1.73 \mathrm{~m}]^{-2}\right)$, calculated with plasma creatinine according to sex, age and ethnicity). Missing values in covariates were imputed by multivariate imputation by chained equations with predictive mean matching using all other covariates and averaged across five iterations. Imputed values were compared against recorded values to assess for aberrations.

\section{Multiplex protein assay}

Blood samples were obtained from individuals instructed to fast overnight, and were then spun down and stored as serum (ULSAM) or EDTA plasma samples (all other cohorts) at $-70^{\circ} \mathrm{C}$ until analysis. The Proseek CVD Multiplex $96 \times 96$ (Olink, Uppsala, Sweden) measures 92 cardiovascular or inflammatory proteins and four internal control samples using the proximity extension assay method (details on quality control, validation and content of the assay are available in electronic supplementary material [ESM] Table 1 and ESM Methods). It has previously been applied to discovering biomarkers for cardiometabolic traits [16-18]. In brief, approximately $10 \mu \mathrm{l}$ of sample were assayed on a 96-well plate, and protein abundance was measured by PCR based on the binding of two specific antibodies for each protein. $\log _{2}$-scaled abundance values adjusted for technical variation with internal controls were transformed to a mean of zero and an SD of 1 . Proteins with $>15 \%$ missing values were excluded. Other missing values were imputed by the lower limit of the detection threshold divided by two. The numbers of missing values are given in ESM Table 2. A total of 12 proteins had $>15 \%$ missing values in at least one cohort and were excluded, leaving 80 proteins for inclusion in the study.

\section{Statistical analysis}

Design The study was divided into two parts, one aimed at biomarker discovery and one at risk prediction (Fig. 1). In part 1, the largest sample, from CARDIPP, was used for discovery, and all other cohorts, combined at the individual level, were used for replication. In part 2, the combined discovery and replication cohorts were randomly split into a $75 \%$ training and a $25 \%$ test set to assess whether the different proteins would improve the prediction of MACE.

Part 1: biomarker discovery Cox proportional hazards regression adjusted for age and sex was used for each protein, with time-to-MACE as outcome. Participants were considered to be at risk until the occurrence of MACE or until the last day of follow-up. An inverse Gaussian frailty effect was included to adjust for heterogeneity between cohorts. The linearity of associations with risk of MACE was assessed by adding a spline term to the linear model (using the pspline function in $\mathrm{R}$ with defaults, and retaining the linearity assumption if the regression $\beta$ coefficient's $p$ value exceeded 0.05 ). Proportional hazards assumptions were assessed in Schoenfeld residual plots and tests of weighted residuals (threshold $p<0.05$ ). The protein assay does not provide standard concentration units, and values were scaled to a mean of zero and an SD of 1. Proteins 
Fig. 1 Study flowchart showing (a) cohorts and (b) further details of the analysis. The combined analysis was adjusted for: sex, current smoking, duration of type 2 diabetes (T2D), BMI, systolic $\mathrm{BP}, \mathrm{HbA}_{1 \mathrm{c}}$, LDL-cholesterol, microalbuminuria, statin use, previous cardiovascular disease (CVD), atrial fibrillation and eGFR. NDR predictors were: age of onset of T2D, T2D duration, total cholesterol/HDLcholesterol, $\mathrm{HbA}_{1 \mathrm{c}}$, systolic $\mathrm{BP}$, BMI, sex, current smoking, microalbuminuria, macroalbuminuria, atrial fibrillation and previous CVD. $\mathrm{PH}$, proportional hazards a

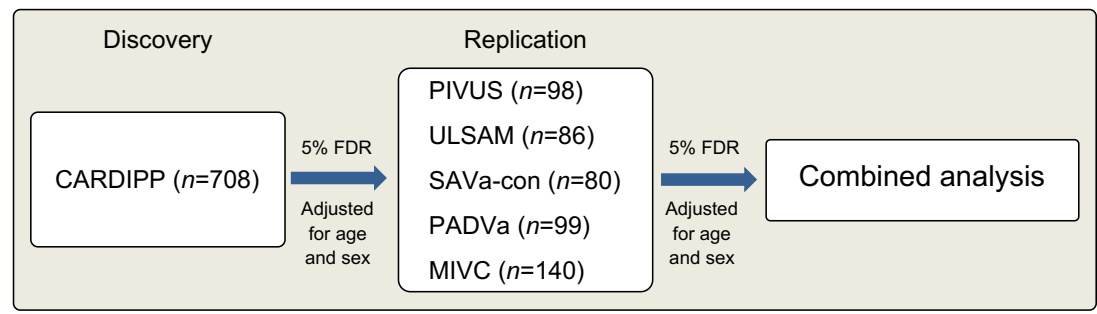

b

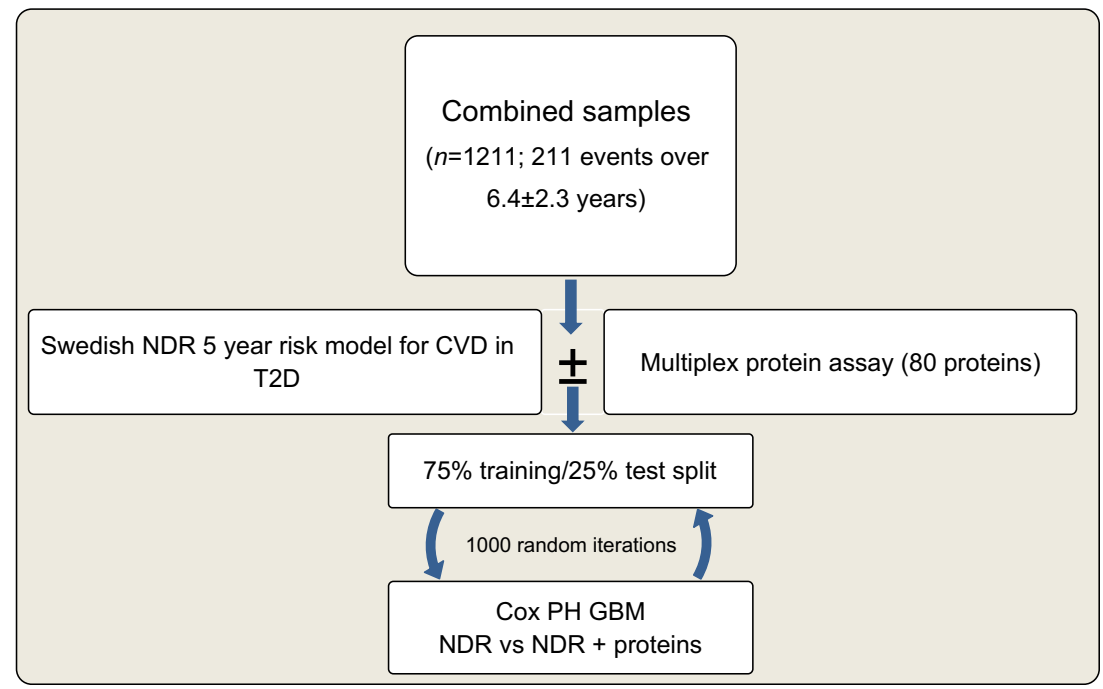

associated below a $5 \%$ false discovery rate (FDR) in the CARDIPP discovery sample were tested in the replication sample, and associations at $<5 \%$ FDR at the replication stage were considered significant. To test for independent associations with MACE, we additionally adjusted for the following established cardiovascular risk factors [25] that were available in the cohorts: atrial fibrillation, BMI, $\mathrm{HbA}_{1 \mathrm{c}}$, LDL-cholesterol, microalbuminuria, systolic blood pressure, sex, smoking, statins, duration of type 2 diabetes, history of cardiovascular disease and eGFR.

Part 2: risk prediction To assess whether adding proteins to established risk factors improved prediction, we tested the performance of the variables included in the NDR risk calculator with and without the protein values. The NDR model (https://www.ndr.nu/IFrameRisk/) [13] was developed in the Swedish NDR to predict 5 year risk of MACE in 30-75-yearolds with type 2 diabetes and comprises age of onset and duration of type 2 diabetes, $\log _{e}$ (total cholesterol/HDLcholesterol), $\log _{e}\left(\mathrm{HbA}_{1 \mathrm{c}}\right), \log _{e}$ (systolic blood pressure), $\log _{e}(\mathrm{BMI})$, sex, current smoker, microalbuminuria, macroalbuminuria, atrial fibrillation and history of cardiovascular disease. This is recommended for evaluating cardiovascular risk in adults with type 2 diabetes by the Swedish National Board of Health and Welfare [26].
We selected the NDR variables for our baseline risk model but used a different statistical approach than that used by Zethelius et al, who developed the NDR calculator [13]. The combined cohorts were randomly split into training (75\%) and test $(25 \%)$ datasets. Cox gradient boosted machine (GBM) learning [27] was applied to the training sample. A baseline model with NDR variables and a baseline-plus-protein model were derived. GBM variables were optimised with regard to model performance (AUC) and complexity as explained in ESM Methods. C statistic, sensitivity and specificity were estimated in the separate test sample. Performance measures and CIs were obtained by bootstrapping in 1000 random iterations. In order to identify a sparse selection of proteins that need to be added to the NDR risk factors to achieve comparable risk discrimination as the whole assay, we used L1regularised lasso Cox regression. We forced the NDR risk factors into the model by setting the penalty variable in the cv.glmnet function in $\mathrm{R}$ to zero, and trained the model by tenfold bootstrapped cross-validation in a random $75 \%$ training sample. The optimum sparse model that minimised the prediction error (selected by lambda.min) was evaluated in the separate $25 \%$ holdout test sample. Analyses were performed in R software version 3.3.2 (https://www.r-project.org/) using the packages survival, nephro, mice, powerSurvEpi, gbm, glmnet, pROC and ggplot2. 


\section{Results}

\section{Sample characteristics}

Figure 1 illustrates the study flow chart, and Table 1 lists the baseline characteristics of all participants. The discovery sample (CARDIPP) recorded 71 MACE events in 708 participants over a mean $( \pm \mathrm{SD})$ of $7.3 \pm 1.8$ years (range $0.1-9.65)$. At a $5 \%$ FDR, we estimated $80 \%$ power to detect an HR of 1.41 per $1 \mathrm{SD}$ change in protein signal. The replication sample combined participants with type 2 diabetes in ULSAM $(n=$ $86 ; 37$ events over $6.8 \pm 3.8$ years), PIVUS ( $n=98 ; 29$ events over $8.1 \pm 2.9$ years), MIVC ( $n=140 ; 38$ events over $2.9 \pm$ 1.2 years), SAVa-control ( $n=80$; ten events over $4.9 \pm$ 1.6 years) and PADVa ( $n=99 ; 26$ events over $4.5 \pm 2.0$ years). The replication set thus included 503 diabetic individuals, 140 of whom experienced a MACE during $5.2 \pm 3.1$ years (range 0.01-12.83), with $80 \%$ power to detect an HR of 1.28 per SD unit of protein. None of the models violated the proportional hazards assumption $(p>0.05)$.

\section{Protein biomarkers associated with risk of MACE}

In the discovery sample, 35 out of 80 proteins were associated with prospective risk of MACE at a 5\% FDR after adjustment for age and sex (ESM Table 3). Eight associations were replicated at $<5 \%$ FDR in the separate replication sample (ESM Table 4). In order to test for associations between biomarkers and MACE independent of established risk factors, we combined all cohorts and tested the eight replicated biomarkers in models adjusted for cardiovascular risk factors. Figure 2 shows the results for the eight biomarkers. In the fully adjusted models, increased levels of the following were associated with incident MACE: matrix metalloproteinase (MMP)-12 (HR per SD increase in protein abundance $1.31,95 \%$ CI 1.17, 1.47); TNF-related apoptosis-inducing ligand receptor 2 (TRAILR2, also known as death receptor 5) (HR 1.44, 95\% CI $1.19,1.74$ ); IL-27 subunit $\alpha$ (IL-27a; HR $1.47,95 \%$ CI 1.21, 1.78); kidney injury molecule (KIM)-1 (HR 1.23, 95\% CI 1.10, 1.36); fibroblast growth factor (FGF)-23 (HR 1.20, 95\% CI, 1.05, 1.37); TNF receptor (TNFR)-1 (HR 1.17, 95\% CI 1.06, 1.28); TNFR-2 (HR 1.37, 95\% CI 1.18, 1.59); and protein S100-A12, also known as extracellular newly identified receptor of advanced glycation end products (RAGE)binding protein (EN-RAGE; HR 1.30, 95\% CI 1.14, 1.48). The exclusion of 12 individuals who had had a haemorrhagic stroke (in MIVC, all five individuals with any type of stroke were excluded as subtypes had not been recorded) had little effect on the effect sizes of the eight biomarkers and replicated the same eight proteins as in the main analysis plus three additional ones (ESM Results, ESM Tables 5-7). A sensitivity analysis with additional adjustment for circulating levels of $\mathrm{N}$ terminal pro-brain natriuretic peptide in those cohorts with available measurements resulted in somewhat increased $p$ values, but essentially left the associations between biomarkers and risk of MACE unchanged (ESM Results, ESM

Table 1 Sample characteristics

\begin{tabular}{|c|c|c|c|c|c|c|}
\hline \multirow[t]{2}{*}{ Variable } & \multicolumn{6}{|l|}{ Cohort } \\
\hline & CARDIPP & ULSAM & PIVUS & MIVC & SAVa-control & PADVa \\
\hline Events/total $N$ & $71 / 708$ & $37 / 86$ & $29 / 98$ & $38 / 140$ & $10 / 80$ & $26 / 99$ \\
\hline Follow-up, years & $7.3 \pm 1.8$ & $6.8 \pm 3.8$ & $8.1 \pm 2.9$ & $2.9 \pm 1.2$ & $4.9 \pm 1.6$ & $4.5 \pm 2.0$ \\
\hline$\%$ women & 34.2 & 0 & 44.9 & 35.6 & 25 & 27.3 \\
\hline Age, years & $60.7 \pm 3.1$ & $77.5 \pm 0.7$ & $70.1 \pm 0.1$ & $62.4 \pm 8.7$ & $68.8 \pm 8.8$ & $68.2 \pm 7.3$ \\
\hline BMI, $\mathrm{kg} / \mathrm{m}^{2}$ & $30.2 \pm 4.7$ & $27.5 \pm 3.5$ & $29.2 \pm 5.4$ & $30.0 \pm 5.2$ & $30.0 \pm 4.0$ & $28.5 \pm 4.0$ \\
\hline $\mathrm{HbA}_{1 \mathrm{c}}, \mathrm{mmol} / \mathrm{mol}$ & $52.9 \pm 11.7$ & $43.9 \pm 14.5$ & NA & $62.6 \pm 19.1$ & $52.3 \pm 13.0$ & $55.3 \pm 12.9$ \\
\hline $\mathrm{HbA}_{1 \mathrm{c}}, \%$ & $7.0 \pm 3.2$ & $6.2 \pm 3.5$ & NA & $7.9 \pm 3.9$ & $6.9 \pm 3.3$ & $7.2 \pm 3.3$ \\
\hline 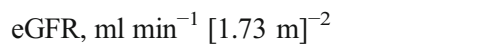 & $78.2 \pm 14.2$ & $64.8 \pm 14.3$ & $65.1 \pm 14.6$ & $19.9 \pm 9.3$ & $66.1 \pm 19.3$ & $53.3 \pm 18.6$ \\
\hline Systolic BP, mmHg & $136.8 \pm 16.5$ & $154.1 \pm 18.5$ & $156.7 \pm 30.0$ & $157.7 \pm 28.8$ & $145.4 \pm 21.0$ & $144.9 \pm 22.5$ \\
\hline Total cholesterol, mmol/1 & $4.3 \pm 0.7$ & $5.2 \pm 0.9$ & $5.1 \pm 0.9$ & $4.8 \pm 1.5$ & $4.5 \pm 1.0$ & $4.8 \pm 1.0$ \\
\hline LDL-cholesterol, mmol/1 & $2.6 \pm 0.8$ & $3.3 \pm 0.8$ & $3.0 \pm 0.9$ & $2.6 \pm 1.2$ & $2.7 \pm 0.9$ & $2.4 \pm 0.9$ \\
\hline HDL-cholesterol, mmol/1 & $1.3 \pm 0.3$ & $1.3 \pm 0.3$ & $1.4 \pm 0.4$ & $1.1 \pm 0.4$ & $1.2 \pm 0.3$ & $1.1 \pm 0.3$ \\
\hline Current smoker, $n(\%)$ & $129(18.2 \%)$ & $6(7.0 \%)$ & $11(11.2 \%)$ & $63(45.0 \%)$ & $3(3.8 \%)$ & $11(11.1 \%)$ \\
\hline History of cardiovascular disease, $n(\%)$ & $76(10.7 \%)$ & $17(19.8 \%)$ & $13(13.3 \%)$ & $62(44.3 \%)$ & $15(18.8 \%)$ & $31(31.3 \%)$ \\
\hline Antihypertensive medication, $n(\%)$ & $343(48.4 \%)$ & $53(61.6 \%)$ & $56(57.1 \%)$ & $138(98.6 \%)$ & $60(75.0 \%)$ & $95(95.6 \%)$ \\
\hline Statin use, $n(\%)$ & $393(55.5 \%)$ & $15(17.4 \%)$ & $25(25.5 \%)$ & $108(77.1 \%)$ & $49(61.3 \%)$ & $84(84.8 \%)$ \\
\hline
\end{tabular}

Continuous variables are given as mean $\pm \mathrm{SD}$ 
Fig. 2 Associations between replicated biomarkers and risk of MACE. Cox regression results in the total sample $(n=1211)$ are given as HR per SD increase in baseline protein levels (error bars denote $95 \%$ CIs), and plotted on a $\log$ scale. Adjustment for age and sex (black symbols and numbers) is compared with additional BMI, $\mathrm{HbA}_{1 \mathrm{c}}$, LDL-cholesterol, microalbuminuria, systolic blood pressure, smoking, statin use, duration of type 2 diabetes, history of cardiovascular disease and eGFR (grey symbols and numbers) adjustment for atrial fibrillation,

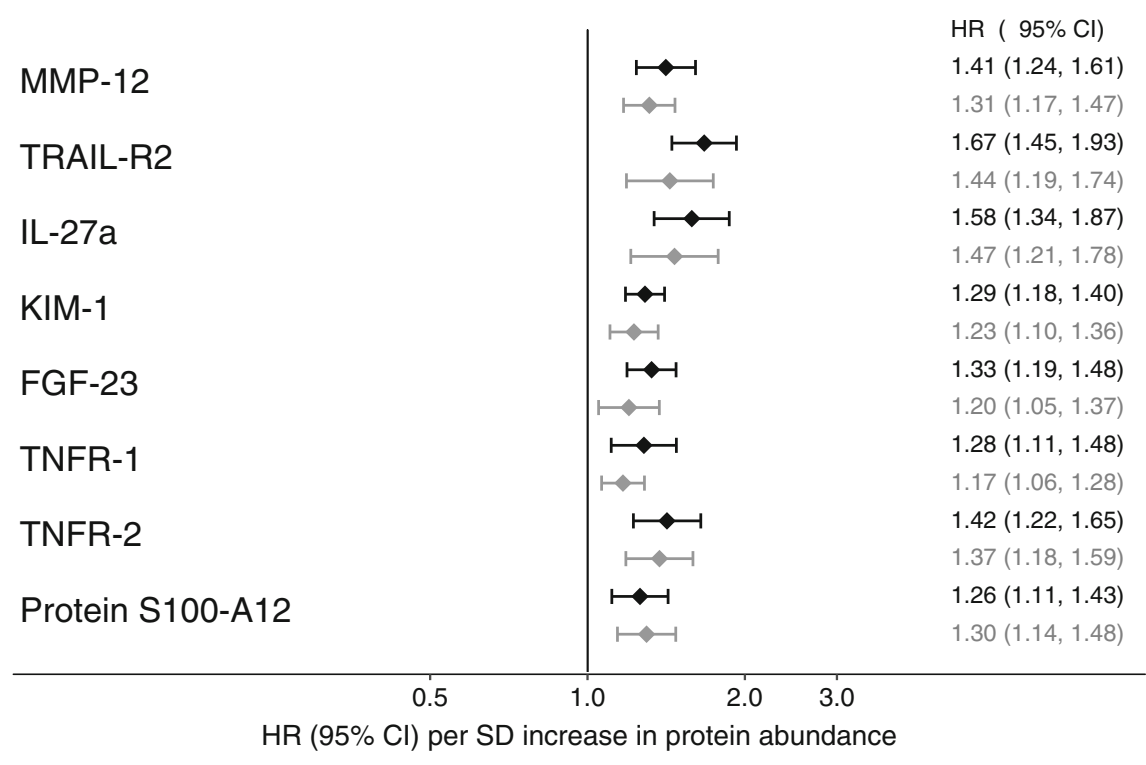

Table 8). Correlations between the biomarkers are shown in ESM Table 9.

\section{Improved risk prediction for MACE in type $\mathbf{2}$ diabetes}

We trained a baseline GBM model including all NDR variables and an NDR-plus-protein model adding all 80 proteins. In the training set of 136 people who experienced a MACE event and 698 people who did not, discrimination improved from $\mathrm{C}=0.738(95 \% \mathrm{CI} 0.735,0.740)$ at baseline to $\mathrm{C}=0.825$ ( $95 \%$ CI $0.824,0.827$ ) with added proteins ( $p$ for difference, $\left.p_{\text {diff }}=3.33 \times 10^{-54}\right)$. Discrimination in the separate test sample of 49 people with and 229 without a MACE event improved from $\mathrm{C}=0.686$ (95\% CI $0.682,0.689)$ to $\mathrm{C}=0.748$ ( $95 \%$ CI $\left.0.746,0.751 ; p_{\text {diff }}=8.48 \times 10^{-21}\right)$. Sensitivity and specificity in the test sample for the upper 50th risk percentile were $70.9 \%$ and $54.0 \%$, respectively, for baseline, and $79.1 \%$ and $55.8 \%$ with added proteins. Sensitivity and specificity for the upper 25th risk percentile in the test set were $48.1 \%$ and $78.9 \%$, respectively, for baseline, and $53.6 \%$ and $80.4 \%$ for added proteins. Lasso Cox regression in the training sample selected 20 proteins in addition to the NDR risk factors. The prediction performance in the independent test sample had a $\mathrm{C}$ statistic of 0.747 (95\% CI $0.653,0.842$ ). A model that included the eight replicated biomarker proteins discovered in part 1 resulted in a C statistic of 0.736 (95\% CI $0.641,0.829)$.

\section{Discussion}

In this prospective multicohort study of adults with type 2 diabetes, we used multiplex proteomics to identify four novel biomarkers associated with prospective risk of a major cardiovascular event independent of potential confounders.
Addition of proteomics data to established risk factors improved the 6 year risk prediction of cardiovascular events.

\section{Novel biomarkers for cardiovascular risk in diabetes}

We identified eight circulating biomarkers, including four novel ones, for incident cardiovascular events after adjustment for established risk factors. Our results replicate previous findings in individuals with type 2 diabetes of associations of increased levels of MMP-12 [17], FGF-23 [28], TNFR-1 and TNFR-2 [29] with incident MACE. For the other four biomarkers, we found no previous studies of prospective associations with MACE in type 2 diabetes, although all have been implicated in cardiometabolic disease in other settings.

Protein S100-A12 (EN-RAGE), the ligand for RAGE, has been associated with incident type 2 diabetes [30] and risk of coronary heart disease [31]. Interaction between RAGE and EN-RAGE triggers an inflammatory cascade, and it has been shown that expression of protein S100-A12 in vascular smooth muscle cells induces oxidative stress, inflammation and vascular remodelling [32].

KIM-1 is mainly expressed in the apical membrane of the renal proximal tubule, and raised circulating levels of KIM-1 are associated with progressive stages of chronic kidney disease in individuals with type 2 diabetes [33, 34]. Associations between raised plasma levels of KIM-1 and adverse cardiovascular risk factors in the general population have recently been reported [35]. Our results in analyses adjusted for kidney function support a potential role of circulating KIM-1 as a cardiovascular risk marker independent of its association with renal function. Our study cannot address the pathogenic mechanisms or potential causality linking KIM-1 to cardiovascular risk in type 2 diabetes, and future experimental studies are indicated. 
TRAIL-R2 is a cell surface receptor for TNF-related apoptosis-inducing ligand (TRAIL), involved in apoptosis. Raised circulating TRAIL-R2 levels have been linked with cerebral atherosclerosis [36] and increased mortality in acute myocardial infarction [37]. Possible mechanisms linking the TRAIL/TRAIL-R2 pathway to atherosclerotic disease involve the endothelial response to cholesterol deposits $[37,38]$ and the composition of circulating fatty acids, as a study in an Alaskan Inuit population found an association between plasma fatty acid levels and genetic variants of the TRAIL-R2 gene TNFRSF10B [39].

IL-27 has complex pro- and anti-inflammatory effects that include direct modification of $\mathrm{CD}^{+}$and $\mathrm{CD} 8^{+} \mathrm{T}$ cells, as well as roles in both innate and antibody-mediated immunity [40]. It has been linked, for instance, to type 1 diabetes [41] and improved atherosclerosis in mice [42], yet functional genetic variants of IL27 were not associated with cardiovascular outcomes in a sample of Chinese individuals [43]. The roles of the four new biomarkers in inflammatory pathways point to an important role of the immune system in cardiovascular pathology in type 2 diabetes. Whether the novel biomarkers might serve as treatment targets remains to be assessed in future studies.

\section{Multiplex proteomics improves prediction beyond established risk factors}

The addition of proteins to the variables included in the NDR risk model significantly improved cardiovascular risk prediction. In our test sample, added biomarkers improved discrimination from $68.6 \%$ to $74.8 \%$, compared with $72.0 \%$ reported in the original publication of the NDR model [13]. The model containing the NDR risk factors plus proteins also improved sensitivity and specificity for the upper half $(79.1 \%$ and $55.8 \%$, compared with $76.2 \%$ and $52.9 \%$, respectively, in the original NDR model [13]) and the upper quarter of predicted risk $(53.6 \%$ and $80.5 \%$, compared with $51.2 \%$ and $77.9 \%$, respectively). Importantly, direct comparisons with the NDR calculator are not possible as we used a different statistical method and study design, as well as a smaller test sample and a somewhat longer follow-up of approximately 6 years. The crucial comparisons are therefore the test set performances in our own sample. Predictor selection with lasso regression retained a subset of 20 proteins in addition to risk factors and achieved a near-identical discrimination performance $(\mathrm{C}=0.747)$ as the model including all 80 proteins. Our results demonstrate that adding proteomics data to known risk factors might aid decision-making for cardiovascular prevention in individuals affected by type 2 diabetes. The protein assay used in this study analyses small sample volumes in under $48 \mathrm{~h}$, making it potentially useful for clinical practice. The accessibility of proteomics platforms is likely to increase in the coming years, and a number of studies have demonstrated how proteomics can discover new biological insights [16-18].

Clinical decisions about whether more aggressive cardiovascular prevention with newer drugs will benefit individuals with type 2 diabetes are difficult, given the progressively smaller benefits, risk of side effects and treatment costs $[7-9,11,14]$. In this study, we demonstrate how a multibiomarker assay can improve risk prediction; future studies in an embedded healthcare setting are indicated to assess the value of '-omics' methods in day-to-day practice. Targeted cardiovascular proteomics might also be useful for streamlining clinical trials of cardiovascular prevention by risk-stratifying participants for cardiovascular prevention, which could lead to improved power to detect clinically meaningful effects and limit expenses [44]. Any application of proteomics with clinical consequences, however, first requires careful validation in future studies.

\section{Strengths and limitations}

Strengths of our study include the prospective community samples, a discovery/replication design and the use of a low sample-volume assay with high-specificity antibody doublets. We limited the risk of overfitting by replicating results in a separate random test subsample and averaging across 1000 iterations, but the bootstrapped CIs have to be interpreted with caution, and our model should be replicated in an independent study. The $\mathrm{C}$ statistic of the baseline model was somewhat lower than expected, which may have led to overoptimistic results after adding proteins. On the other hand, the $\mathrm{C}$ statistic is usually rather insensitive to added predictors, and we showed convincing improvement [45]. Limitations include the moderate sample size, lack of power to assess the components of MACE as separate outcomes, and failure of 12 proteins in quality control because of missing values. Generalisability is limited to middle-aged to elderly adults (30-77 years of age). Analyses accounted for heterogeneity between cohorts and, rather than limiting variability and effective sample size by tightening the inclusion criteria, we attempted to increase external validity by including a broader range of individuals with type 2 diabetes that would reflect clinical reality.

\section{Conclusion}

We found that a high-throughput multiprotein assay for presumptive disease markers can identify novel biomarkers and improve the identification of individuals with type 2 diabetes at highest risk of a cardiovascular event. Larger clinic-based studies are needed to assess the value of multiplex proteomics in a healthcare context. 
Data availability The authors report that, for approved reasons, some access restrictions apply to the data underlying this study. Phenotypes from ULSAM, PIVUS, CARDIPP, MIVC, SAVa-control and PADVa are not publicly available for ethical reasons, as agreed upon by participating volunteers in their informed consent. Data are available on request for researchers who meet the criteria for confidential data access. Data from the ULSAM study are available from the ULSAM steering committee (http://www.pubcare.uu.se/ulsam/Database; contact: V. Giedraitis, vilmantas.giedraitis@pubcare.uu.se). Data from the PIVUS study are available from the PIVUS steering committee (http://www.medsci.uu. se/pivus/; contact: lars.lind@medsci.uu.se). Data from the MIVC study are available from the MIVC steering committee (contact: A. Cordeiro, accordeirojr@uol.com.br). Data requests for the PADVa/SAVa study should be addressed to the steering group (https://savastudy.se/ coworkers/; contact: P. Hedberg, par.o.hedberg@regionvastmanland.se). Data requests in the CARDIPP study should be addressed to the steering committee (details: https://clinicaltrials.gov/ct2/show/NCT01049737; contact: C. J. Östgren, carl.johan.ostgren@liu.se).

Funding This work was supported by the European Union Horizon 2020 project (grant 634869), the Swedish Research Council (grants 2012 2215, 2015-03477), Landstinget Dalarna (Falun, Sweden), Dalarna University (Falun, Sweden), Sparbanksstiftelsen Nya (grants 552, 693, 932, 2297), Region Västmanland (Västerås, Sweden), the Swedish Medical Association and the Swedish Heart-Lung Foundation (grant 20150429).

Duality of interest EI is a scientific advisor for Olink Proteomics for projects unrelated to the present study. The company had no influence over the design, analysis or interpretation of data in the present study, and did not provide any funding for the study. JÄ has received lecturing fees from AstraZeneca unrelated to the present project. The authors report that there are no other duality of interests associated with their contribution to this manuscript.

Contribution statement $\mathrm{CN}$ and JÄ designed the study; $\mathrm{CN}$ analysed the data and wrote the manuscript draft. MA contributed to data analysis. ACCa, CJÖ, FHN, MA, TFe, JS, JJC, JL, PH, EH, ACCo, VG, LL, EI, $\mathrm{TFa}$ and $\mathrm{JÄ}$ critically revised the study design and manuscript. CJÖ, FHN, JS, EH, ACCo, LL, EI, TFa and JÄ obtained funding for the study. $\mathrm{JÄ}$ is the guarantor of the study. All authors critically revised the manuscript. All authors have approved the final version to be published.

Open Access This article is distributed under the terms of the Creative Commons Attribution 4.0 International License (http:// creativecommons.org/licenses/by/4.0/), which permits unrestricted use, distribution, and reproduction in any medium, provided you give appropriate credit to the original author(s) and the source, provide a link to the Creative Commons license, and indicate if changes were made.

\section{References}

1. International Diabetes Federation (2017) IDF Diabetes Atlas, 8th edn. International Diabetes Federation, Brussels

2. Gregg EW, Zhuo X, Cheng YJ, Albright AL, Narayan KMV, Thompson TJ (2014) Trends in lifetime risk and years of life lost due to diabetes in the USA, 1985 to 2011: a modelling study. Lancet Diabetes Endocrinol 2:867-874

3. Haffner SM, Lehto S, Rönnemaa T, Pyörälä K, Laakso M (1998) Mortality from coronary heart disease in subjects with type 2 diabetes and in nondiabetic subjects with and without prior myocardial infarction. N Engl J Med 339:229-234
4. Berry C, Tardif J-C, Bourassa MG (2007) Coronary heart disease in patients with diabetes. J Am Coll Cardiol 49:631-642

5. Pierre-Louis B, Aronow WS, Palaniswamy C et al (2009) Obstructive coronary artery disease in high-risk diabetic patients with and without atrial fibrillation. Coron Artery Dis 20:91-93

6. Sattar N, Preiss D, Murray HM et al (2010) Statins and risk of incident diabetes: a collaborative meta-analysis of randomised statin trials. Lancet 375:735-742

7. Ference BA, Robinson JG, Brook RD et al (2016) Variation in PCSK9 and HMGCR and risk of cardiovascular disease and diabetes. N Engl J Med 375:2144-2153

8. Sabatine MS, Giugliano RP, Wiviott SD et al (2015) Efficacy and safety of evolocumab in reducing lipids and cardiovascular events. N Engl J Med 372:1500-1509

9. Neal B, Perkovic V, Mahaffey KW et al (2017) Canagliflozin and cardiovascular and renal events in type 2 diabetes. N Engl J Med 377:644-657

10. Gandra SR, Villa G, Fonarow GC et al (2016) Cost-effectiveness of LDL-C lowering with evolocumab in patients with high cardiovascular risk in the United States. Clin Cardiol 39:313-320

11. Korman M, Wisloff T (2018) Modelling the cost-effectiveness PCSK9 inhibitors vs. ezetimibe through LDL-C reductions in a Norwegian setting. Eur Heart Journal Cardiovasc Pharmacother 4: $15-22$

12. Muntner P, Whelton PK (2017) Using predicted cardiovascular disease risk in conjunction with blood pressure to guide antihypertensive medication treatment. J Am Coll Cardiol 69:2446-2456

13. Zethelius B, Eliasson B, Eeg-Olofsson K, Svensson AM, Gudbjornsdottir S, Cederholm J (2011) A new model for 5-year risk of cardiovascular disease in type 2 diabetes, from the Swedish National Diabetes Register (NDR). Diabetes Res Clin Pract 93: 276-284

14. Schiele F, Ecarnot F, Chopard R (2017) Coronary artery disease: risk stratification and patient selection for more aggressive secondary prevention. Eur J Prev Cardiol 24:88-100

15. Hoefer IE, Steffens S, Ala-Korpela M et al (2015) Novel methodologies for biomarker discovery in atherosclerosis. Eur Heart J 36: 2635-2642

16. Nowak C, Sundstrom J, Gustafsson S et al (2016) Protein biomarkers for insulin resistance and type 2 diabetes risk in two large community cohorts. Diabetes 65:276-284

17. Goncalves I, Bengtsson E, Colhoun HM et al (2015) Elevated plasma levels of MMP-12 are associated with atherosclerotic burden and symptomatic cardiovascular disease in subjects with type 2 diabetes. Arterioscler Thromb Vasc Biol 35:1723-1731

18. Ljungberg J, Janiec M, Bergdahl IA et al (2018) Proteomic biomarkers for incident aortic stenosis requiring valvular replacement. Circulation https://doi.org/10.1161/CIRCULATIONAHA.117. 030414

19. Looker HC, Colombo M, Agakov F et al (2015) Protein biomarkers for the prediction of cardiovascular disease in type 2 diabetes. Diabetologia 58:1363-1371

20. Dahlen EM, Lanne T, Engvall J et al (2009) Carotid intima-media thickness and apolipoprotein B/apolipoprotein A-I ratio in middleaged patients with type 2 diabetes. Diabet Med 26:384-390

21. Lind L, Fors N, Hall J, Marttala K, Stenborg A (2006) A comparison of three different methods to determine arterial compliance in the elderly: the Prospective Investigation of the Vasculature in Uppsala Seniors (PIVUS) study. J Hypertens 24:1075-1082

22. Hedstrand H (1975) A study of middle-aged men with particular reference to risk factors for cardiovascular disease. Upps J Med Sci Suppl 19:1-61

23. Hedberg P, Hammar C, Selmeryd J et al (2014) Left ventricular systolic dysfunction in outpatients with peripheral atherosclerotic vascular disease: prevalence and association with location of arterial disease. Eur J Heart Fail 16:625-632 
24. Cordeiro AC, Lindholm B, Sousa MG et al (2014) Reliability of electrocardiographic surrogates of left ventricular mass in patients with chronic kidney disease. J Hypertens 32:439-445

25. World Health Organization (2007) Prevention of cardiovascular disease. Guidelines for assessment and management of cardiovascular risk. Geneva: WHO

26. Socialstyrelsen (2017) Nationella riktlinjer för diabetesvård. Stöd för styrning och ledning. Stockholm: Socialstyrelsen

27. Friedman JH (2002) Stochastic gradient boosting. Comput Stat Data Anal 38:367-378

28. Tunon J, Fernandez-Fernandez B, Carda R et al (2016) Circulating fibroblast growth factor-23 plasma levels predict adverse cardiovascular outcomes in patients with diabetes mellitus with coronary artery disease. Diabetes Metab Res Rev 32:685-693

29. Carlsson AC, Ostgren CJ, Nystrom FH et al (2016) Association of soluble tumor necrosis factor receptors 1 and 2 with nephropathy, cardiovascular events, and total mortality in type 2 diabetes. Cardiovasc Diabetol 15:40

30. Brahimaj A, Ligthart S, Ghanbari M et al (2017) Novel inflammatory markers for incident pre-diabetes and type 2 diabetes: the Rotterdam Study. Eur J Epidemiol 32:217-226

31. Ligthart S, Sedaghat S, Ikram MA, Hofman A, Franco OH, Dehghan A (2014) EN-RAGE: a novel inflammatory marker for incident coronary heart disease. Arterioscler Thromb Vasc Biol 34: 2695-2699

32. Hofmann Bowman M, Wilk J, Heydemann A et al (2010) S100A12 mediates aortic wall remodeling and aortic aneurysm. Circ Res 106: $145-154$

33. Coca SG, Nadkarni GN, Huang Y et al (2017) Plasma biomarkers and kidney function decline in early and established diabetic kidney disease. J Am Soc Nephrol 28:2786-2793

34. Nowak N, Skupien J, Smiles AM et al (2018) Markers of early progressive renal decline in type 2 diabetes suggest different implications for etiological studies and prognostic test development. Kidney Int 93:1198-1206

35. Egli P, Aeschbacher S, Bossard M et al (2018) Relationships of kidney injury molecule-1 with renal function and cardiovascular risk factors in the general population. Clin Chim Acta 478:13-17

36. Pan X, Pang M, Ma A et al (2015) Association of TRAIL and its receptors with large-artery atherosclerotic Stroke. PLoS One 10: e0136414

37. Skau E, Henriksen E, Wagner P, Hedberg P, Siegbahn A, Leppert J (2017) GDF-15 and TRAIL-R2 are powerful predictors of longterm mortality in patients with acute myocardial infarction. Eur J Prev Cardiol 24:1576-1583

38. Pang J, Poulter EB, Bell DA et al (2015) Frequency of familial hypercholesterolemia in patients with early-onset coronary artery disease admitted to a coronary care unit. J Clin Lipidol 9:703-708

39. Voruganti VS, Cole SA, Ebbesson SO et al (2010) Genetic variation in APOJ, LPL, and TNFRSF10B affects plasma fatty acid distribution in Alaskan Eskimos. Am J Clin Nutr 91:1574-1583

40. Yoshida H, Hunter CA (2015) The immunobiology of interleukin27. Annu Rev Immunol 33:417-443

41. Wang R, Han G, Wang J et al (2008) The pathogenic role of interleukin-27 in autoimmune diabetes. Cell Mol Life Sci 65: $3851-3860$

42. Hirase T, Hara H, Miyazaki Y et al (2013) Interleukin 27 inhibits atherosclerosis via immunoregulation of macrophages in mice. Am J Phys Heart Circ Phys 305:H420-H429

43. Fan Q, Nie S, Li S et al (2016) Analysis of the genetic association between IL27 variants and coronary artery disease in a Chinese Han population. Sci Rep 6:25782

44. Woodcock J, LaVange LM (2017) Master protocols to study multiple therapies, multiple diseases, or both. N Engl J Med 377:62-70

45. Cook NR (2007) Use and misuse of the receiver operating characteristic curve in risk prediction. Circulation 115:928-935 